\title{
Transcorrelated Density Matrix Renormalization Group
}

\author{
Alberto Baiardi ${ }^{1, a)}$ and Markus Reiher ${ }^{1, b}$ ) \\ ETH Zürich, Laboratorium für Physikalische Chemie, Vladimir-Prelog-Weg 2, \\ 8093 Zürich, Switzerland.
}

(Dated: September 30, 2020)

\begin{abstract}
We introduce the transcorrelated Density Matrix Renormalization Group (tcDMRG) theory for the efficient approximation of the energy for strongly correlated systems. tcDMRG encodes the wave function as a product of a fixed Jastrow or Gutzwiller correlator and a matrix product state. The latter is optimized by applying the imaginarytime variant of time-dependent (TD) DMRG to the non-Hermitian transcorrelated Hamiltonian. We demonstrate the efficiency of tcDMRG at the example of the two-dimensional Fermi-Hubbard Hamiltonian, a notoriously difficult target for the DMRG algorithm, for different sizes, occupation numbers, and interaction strengths. We demonstrate fast energy convergence of tcDMRG, which indicates that tcDMRG could increase the efficiency of standard DMRG beyond quasi-monodimensional systems and provides a generally powerful approach toward the dynamic correlation problem of DMRG.
\end{abstract}

\footnotetext{
a) Electronic mail: alberto.baiardi@phys.chem.ethz.ch

b)Electronic mail: markus.reiher@phys.chem.ethz.ch
} 


\section{INTRODUCTION}

Recent years have witnessed a growing interest in efficient configuration interaction (CI) based algorithms, such as full-CI Quantum Monte Carlo (FCIQMC) algorithm, ${ }^{1,2}$ different formulations of selected CI, ${ }^{3-7}$ and tensor-network-based approaches, such as the density matrix renormalization group (DMRG). ${ }^{8-22}$ These advances allow for the calculation of full-CI (and complete active space-CI) energies for Hamiltonians with up to about 100 orbitals, ${ }^{23,24}$, thereby extending the range of methods aiming at an accurate treatment of static correlation effects. However, to develop efficient and reliable options for assessing the then still missing dynamical correlation effects remains to be a major challenge. Approaches based on perturbation theory lead to a steep increase of the computational cost of both tensornetwork-based approaches ${ }^{25-35}$ and of selected CI algorithms ${ }^{36-38}$ due to the large size of the virtual orbital space and the unfavorable scaling of the number of elements of higher-order reduced density matrices. Alternative strategies, such as the combination of such methods with density functional theory (DFT), ${ }^{39-43}$ have been explored. However, they depend on the choice of a density functional and their ultimate accuracy has not been well established yet.

An alternative solution to the dynamical correlation problem is provided by explicitlycorrelated methods ${ }^{44-47}$ that add to the wave function parametrization terms depending explicitly on inter-electronic distances. In this way, the energy convergence with basis set size is faster, because of better accounting for the consequences of the singular Coulomb interactions of the electrons at short range. As a consequence, accurate results are obtained with smaller orbital spaces. As a side remark, we note that the handling of short-range dynamic correlation by short-range density functionals has also shown to regularize active orbital spaces making them more compact and stable with respect to changes in the active space. ${ }^{41}$ So-called F12-based algorithms are now routinely applied in single-reference theories

such as Møller-Plesset perturbation theory and coupled cluster theory, ${ }^{48,49}$ but their multireference generalizations are much less explored. ${ }^{50,51}$

An alternative is the transcorrelation approach originally introduced by Boys and Handy. ${ }^{52,53}$ Transcorrelated methods parametrize the wave function as a product of a CI-like wave function and a fixed Jastrow factor. ${ }^{54}$ The latter is revolved from the wavefunction to the definition of the Hamiltonian by similarity transformation. The former 
can then be optimized by applying standard quantum-chemical methods (such as Davidson subspace diagonalization) to the resulting similarity-transformed Hamiltonian known as the transcorrelated Hamiltonian. However, two factors have impeded so-far a widespread use of these approaches to quantum chemistry. First, the transcorrelated Hamiltonian contains three-body interactions, which are technically hard to include in common quantum-chemical methods as they require the implementation of new integrals over Gaussian basis functions. Second, the transcorrelated Hamiltonian is a non-Hermitian operator, which prevents a trivial application of any variational method. Even though several strategies have been proposed to overcome this second problem, ${ }^{55-57}$ none has proven to be a reliable alternative to F12-based schemes.

Luo and Alavi showed ${ }^{58}$ that methods based on imaginary-time evolution, such as FCIQMC, can be straightforwardly applied to non-Hermitian Hamiltonians. Based on this idea, they showed that the convergence of FCIQMC is much faster when applied to transcorrelated Hamiltonians, both for the Fermi-Hubbard ${ }^{59}$ and for the non-relativistic electronic Hamiltonians. ${ }^{60}$ The same idea has been recently exploited in the design of quantumcomputing algorithms. ${ }^{61,62}$ Inspired by the success of the transcorrelated FCIQMC theory, we introduce here the transcorrelated DMRG (tcDMRG). tcDMRG encodes the eigenvector of the transcorrelated Hamiltonian as a matrix product state (MPS) and optimizes it with imaginary-time time-dependent DMRG. Among the various TD-DMRG theories proposed in the literature, ${ }^{63}$ we rely in the present work on the tangent-space formulation of TDDMRG $^{64-66}$ that can support arbitrarily complex Hamiltonians, such as the transcorrelated one.

We apply tcDMRG to the calculation of the ground-state energy of the transcorrelated two-dimensional Fermi-Hubbard Hamiltonian that has a closed-form analytic expression and is therefore the ideal first test-case for tcDMRG. We show that the energy convergence of tcDMRG is much faster than for standard DMRG in both weak and strong correlation regimes. This suggests that the long-ranged interactions that make DMRG inefficient can be effectively reduced by similarity transformation. The eigenvector of the resulting nonHermitian Hamiltonian can be effectively represented as a low-entanglement wave function and optimized with imaginary-time TD-DMRG. 


\section{TRANSCORRELATED DMRG THEORY}

\section{A. Two-dimensional Fermi-Hubbard model}

The Fermi-Hubbard (FH) Hamiltonian for a two-dimensional lattice of width $W$, height $H, N_{\alpha}$ spin-up and $N_{\beta}$ spin-down electrons reads:

$$
\mathcal{H}_{\mathrm{FH}}^{(\mathrm{r})}=-t \sum_{\langle\boldsymbol{i}, \boldsymbol{j}\rangle} \sum_{\sigma} a_{\boldsymbol{i}, \sigma}^{\dagger} a_{\boldsymbol{j}, \sigma}+U \sum_{\boldsymbol{i}} n_{\alpha, \boldsymbol{i}} n_{\beta, \boldsymbol{i}},
$$

where $\boldsymbol{i}=\left(i_{x}, i_{y}\right)$, with $0 \leq i_{x} \leq H$ and $0 \leq i_{y} \leq W$, and $\langle\boldsymbol{i}, \boldsymbol{j}\rangle$ denotes a sum over neighboring sites of the lattice. The first term is referred to as "hopping" term, while the second one is the "interaction" term, and the ratio between $t$ and $U$ defines the relative

magnitude of the two terms. Moreover, $a_{\boldsymbol{i}, \sigma}^{\dagger}$ is the creation operator for orbital $\boldsymbol{i}$ with spin $\sigma$, while $a_{\boldsymbol{i}, \sigma}$ and $n_{\sigma, \boldsymbol{i}}$ are the corresponding annihilation and number operators, respectively. The multireference character of the ground state of Eq. (1) increases with the $U / t$ ratio, and therefore large $U / t$ values correspond to a strongly correlated regime.

The Hamiltonian defined in Eq. (1) is usually known as the real-space ('r') representation of the Fermi-Hubbard Hamiltonian, since each orbital corresponds to a specific site of the lattice. The real-space Hamiltonian of Eq. (1) can be expressed in a momentum-space ('m') representation (referred to as $\boldsymbol{k}$-space representation in the following) by a unitary transformation of the creation operator $a_{i, \sigma}$ as follows:

$$
c_{\boldsymbol{k}, \sigma}=\frac{1}{\sqrt{W L}} \sum_{i_{x}=1}^{W} \sum_{i_{y}=1}^{H} e^{\mathrm{i} \boldsymbol{k} \cdot \boldsymbol{i}} a_{\boldsymbol{i}, \sigma}
$$

where $\boldsymbol{k}=\left(k_{x}, k_{y}\right)$ with $-W / 2 \leq k_{x} \leq W / 2$ and $-H / 2 \leq k_{y} \leq H / 2$. The momentum-space representation of the Fermi-Hubbard Hamiltonian, obtained by combing Eqs. (2) and (1), reads:

$$
\mathcal{H}_{\mathrm{FH}}^{(\mathrm{m})}=-t \sum_{\boldsymbol{k}, \sigma} \epsilon_{\boldsymbol{k}} n_{\boldsymbol{k}, \sigma}+U \sum_{\boldsymbol{p}, \boldsymbol{q}, \boldsymbol{k}, \sigma} c_{\boldsymbol{p}-\boldsymbol{k}, \sigma}^{\dagger} c_{\boldsymbol{q}+\boldsymbol{k}, \bar{\sigma}}^{\dagger} c_{\boldsymbol{q}, \bar{\sigma}} c_{\boldsymbol{p}, \sigma}
$$

Compared to Eq. (1), the hopping term has a simpler diagonal form, while the interaction term becomes more complex since it includes strings of four potentially different secondquantization operators. Note that the Hamiltonian defined Eq. (3) has the same structure as the quantum chemical Hamiltonian in electronic structure theory. The only major difference 
is that all interaction terms of Eq. (3) are scaled by the same factor (i.e., $U$ ), whereas such factor will be different for each combination of orbitals in the quantum chemical Hamiltonian.

It is known that, in the weakly correlated regime ( small $U / t)$, the ground state of the momentum-space Fermi-Hubbard Hamiltonian is mostly single-reference, whereas that of the real-space Hamiltonian is multi-reference. For this reason, methods that approximate the ground-state wave function based on a single reference determinant, such as configuration interaction ones, are more efficient when applied to Eq. (3) than to Eq. (1). However, in the strong correlation regime, the ground-state wave functions of both display a strong multireference character. To tame such correlation effects, it is convenient to parametrize the ground-state wave function of the Fermi-Hubbard Hamiltonian with a Jastrow-like ansatz as the following on:

$$
\left|\Phi_{\mathrm{tc}}\right\rangle=e^{\tau}|\Phi\rangle
$$

with

$$
\tau=J \sum_{\boldsymbol{l}} n_{\boldsymbol{l}, \alpha} n_{\boldsymbol{l}, \beta}
$$

$|\Phi\rangle$ is parametrized with standard quantum-chemical methods, such as Hartree-Fock (HF) or full-CI ${ }^{59,67}$ (note that Eq. (4) applies to both Hamiltonian representations). The Jastrow factor expressed in the second-quantization space is also known as Gutzwiller correlator, and is uniquely defined by the single parameter $J$. Inspired by Ref. 59 , where $|\Phi\rangle$ is parametrized as a full-CI wave function, we here encode $|\Phi\rangle$ as a matrix product state $\left(\Phi_{\text {MPS }}\right)$.

The Jastrow parameter $J$ and the wave function $|\Phi\rangle$ can in principle be optimized simultaneously to minimize the energy functional variationally. However, for a given $J$ value, the optimal $|\Phi\rangle$ wave function is obtained as the right eigenfunction of the following similarity-transformed Hamiltonian, known as the transcorrelated Hamiltonian:

$$
\mathcal{H}_{\mathrm{tcFH}}\left|\Phi_{\mathrm{tc}}\right\rangle=\left(e^{-\tau} \mathcal{H}_{\mathrm{FH}} e^{\tau}\right)\left|\Phi_{\mathrm{tc}}\right\rangle=E_{\mathrm{tc}}\left|\Phi_{\mathrm{tc}}\right\rangle
$$

A closed-form expression for $\mathcal{H}_{\mathrm{tcFH}}$ is obtained by evaluating $e^{-\tau} \mathcal{H}_{\mathrm{FH}} e^{\tau}$ with a BakerCampbell-Hausdorff formula. This leads to a many-body expansion that, for the specific definition of the correlator given in Eq. (5), truncates at low order. The transcorrelated real-space Fermi-Hubbard Hamiltonian reads 


$$
\begin{aligned}
\mathcal{H}_{\mathrm{tcFH}}^{(\mathrm{r})}= & -t \sum_{\langle\boldsymbol{i}, \boldsymbol{j}\rangle} \sum_{\sigma} a_{\boldsymbol{i}, \sigma}^{\dagger} a_{\boldsymbol{j}, \sigma}+U \sum_{\boldsymbol{i}} n_{\alpha, \boldsymbol{i}} n_{\beta, \boldsymbol{i}}+2 t \sum_{\langle\boldsymbol{i}, \boldsymbol{j}\rangle} \sum_{\sigma} a_{\boldsymbol{i}, \sigma}^{\dagger} a_{\boldsymbol{j}, \sigma} \cosh (J) n_{\boldsymbol{i}, \bar{\sigma}} n_{\boldsymbol{j}, \bar{\sigma}} \\
& -t\left(e^{J}-1\right) \sum_{\langle\boldsymbol{i}, \boldsymbol{j}\rangle} \sum_{\sigma} a_{\boldsymbol{i}, \sigma}^{\dagger} a_{\boldsymbol{j}, \sigma} n_{\boldsymbol{j}, \bar{\sigma}}-t\left(e^{-J}-1\right) \sum_{\langle\boldsymbol{i}, \boldsymbol{j}\rangle} \sum_{\sigma} a_{\boldsymbol{i}, \sigma}^{\dagger} a_{\boldsymbol{j}, \sigma} n_{\boldsymbol{i}, \bar{\sigma}}
\end{aligned}
$$

Eq. (7) was first derived in Ref. 68, and later applied to Hartree-Fock wave functions, ${ }^{67}$ Monte Carlo-based methods, ${ }^{69}$ and FCIQMC. ${ }^{59}$ The momentum-space counterpart of Eq. (7) was first derived in Ref. 59 and reads:

$$
\begin{aligned}
\mathcal{H}_{\mathrm{tcFH}}^{(\mathrm{m})}= & -t \sum_{\boldsymbol{k}, \sigma} \epsilon_{\boldsymbol{k}} n_{\boldsymbol{k}, \sigma}+\sum_{\boldsymbol{p}, \boldsymbol{q}, \boldsymbol{k}, \sigma}\left(\frac{U}{2}-t\left[\left(e^{J}-1\right) \epsilon_{\boldsymbol{p}-\boldsymbol{k}}+\left(e^{-J}-1\right) \epsilon_{\boldsymbol{p}}\right]\right) c_{\boldsymbol{p}-\boldsymbol{k}, \sigma}^{\dagger} c_{\boldsymbol{q}+\boldsymbol{k}, \bar{\sigma}}^{\dagger} c_{\boldsymbol{q}, \bar{\sigma}} c_{\boldsymbol{p}, \sigma} \\
& +2 t \frac{\cosh (J)-1}{W^{2} H^{2}} \sum_{\boldsymbol{p}, \boldsymbol{q}, \boldsymbol{s}, \boldsymbol{k}, \boldsymbol{k}^{\prime}, \sigma} \epsilon_{\boldsymbol{p}-\boldsymbol{k}+\boldsymbol{k}^{\prime}} c_{\boldsymbol{p}-\boldsymbol{k}, \sigma}^{\dagger} c_{\boldsymbol{q}+\boldsymbol{k}^{\prime}, \bar{\sigma}}^{\dagger} c_{\boldsymbol{s}+\boldsymbol{k}-\boldsymbol{k}^{\prime}, \bar{\sigma}}^{\dagger} c_{\boldsymbol{s}, \bar{\sigma}} c_{\boldsymbol{q}, \bar{\sigma}} c_{\boldsymbol{p}, \sigma}
\end{aligned}
$$

Correlation effects in the two-dimensional Fermi-Hubbard model are determined not only by the $U / t$ ratio, but also by the Hamiltonian boundary conditions. Following Ref. 59, we rely on full periodic boundary conditions, i.e. the hopping term in Eq. 1 couples sites with $i_{x}=j_{x}, i_{y}=0, i_{y}=W$, and with $i_{y}=j_{y}, i_{x}=0$, and $i_{x}=H$. Alternative choices are open boundary conditions, where no periodicity is imposed, and so-called cylindrical boundary conditions, where periodicity is imposed along only one of the two dimensions. ${ }^{70}$ A hybrid real/momentum space representation of the two-dimensional FermiHubbard Hamiltonian ${ }^{71,72}$ based on cylindrical boundary conditions can target lattices with up to $H=32, W=6$. Even though such sizes cannot be targeted with the setup adopted here, we rely on periodic boundary conditions to enhance correlation effects and, therefore, challenge the accuracy of tcDMRG.

\section{B. Imaginary-time DMRG optimization}

Both Eq. (7) and Eq. (8) define non-Hermitian operators, and this impedes a variational optimization of $E_{\mathrm{tc}}$ with standard DMRG. However, Alavi and co-workers ${ }^{58}$ proved that the right eigenvector of $\mathcal{H}_{\mathrm{tc}}$, both in real- and momentum-space representation, can be optimized by imaginary-time evolution, i.e. 


$$
\left|\Phi_{\mathrm{tc}}\right\rangle=\lim _{t \rightarrow+\infty} e^{-\mathcal{H}_{\mathrm{tc}} t}\left|\Phi_{\text {guess }}\right\rangle
$$

where $\left|\Phi_{\text {guess }}\right\rangle$ is a guess wave function such that $\left\langle\Phi_{\mathrm{tc}} \mid \Phi_{\text {guess }}\right\rangle \neq 0$. Instead of evaluating the limit, Eq. (9) is often implemented by applying repeatedly the time-evolution operator $e^{-\mathcal{H}_{\mathrm{tc}} \Delta t}$ for a finite time step $\Delta t$, until convergence. Luo and Alavi solved Eq. (9) stochastically with FCIQMC, ${ }^{58}$ by representing $\left|\Phi_{\mathrm{tc}}\right\rangle$ as an ensemble of discrete walkers. In the present work, we encode instead $\left|\Phi_{\mathrm{tc}}\right\rangle$ as an MPS $\left|\Phi_{\text {MPS }}^{\mathrm{tc}}\right\rangle$ and optimize it with the imaginary-time version of the density matrix renormalization group (DMRG) theory (iTD-DMRG). The solution of Eq. (9) can be obtained by taking the $t \rightarrow+\infty$ limit of the solution of the following differential equation

$$
\frac{\partial\left|\Phi_{\mathrm{MPS}}^{\mathrm{tc}}(t)\right\rangle}{\partial t}=-\mathcal{H}_{\mathrm{tc}}\left|\Phi_{\mathrm{MPS}}^{\mathrm{tc}}(t)\right\rangle
$$

where the wave function $\left|\Phi_{\mathrm{MPS}}^{\mathrm{tc}}(t)\right\rangle$ is expressed as an MPS,

$$
\left|\Phi_{\mathrm{MPS}}^{\mathrm{tc}}(t)\right\rangle=\sum_{\boldsymbol{\sigma}} \sum_{\boldsymbol{m}} M_{1, m_{1}}^{\sigma_{1}}(t) M_{m_{1}, m_{2}}^{\sigma_{2}}(t) \cdots M_{m_{L-1}, 1}^{\sigma_{L}}(t)|\boldsymbol{\sigma}\rangle .
$$

In an MPS, the CI tensor is replaced by a product of $L$ three-dimensional tensors, one per site $i\left(M_{m_{i-1}, m_{i}}^{\sigma_{i}}\right)$. From a numeric analysis perspective, Eq. (11) is obtained from a standard CI expansion by replacing the CI tensor with its tensor-train factorization. The index $\sigma_{i}$ in $\boldsymbol{\sigma}=\left(\sigma_{1}, . ., \sigma_{L}\right)$ (usually referred to as physical index) runs over all possible occupations for the $i$-th orbital (referred to as "site" in DMRG terminology). The maximum dimension for the $m_{i-1}$ and $m_{i}$ indices is the "bond dimension" and tunes the accuracy of approximating a CI wave function as in Eq. (11). DMRG will be efficient if an accurate representation of the wave function can be obtained with low $m$ values. The area law ${ }^{73}$ ensures that this is the case for the ground-state wave function of short-ranged Hamiltonians. In the DMRG context, "short-range" means that it exists a sorting of the orbitals such that only neighboring ones interact. This is not the case of the two-dimensional Fermi-Hubbard model, neither in real-space, due to the off-diagonal hopping terms, nor in momentum-space, where the potential-energy is long-range. However, as Alavi showed that the ground state of the Fermi-Hubbard Hamiltonian can be encoded as in Eq. (4) by expressing $|\Phi\rangle$ as a compact CI expansion, we aim here at showing that $|\Phi\rangle$ can be efficiently encoded as an MPS with a low bond dimension. 
Eq. (10) cannot be solved exactly by fixing the bond dimension $m$ of the MPS at all times because the bond dimension of the MPS representation of $\mathcal{H} \Phi_{\text {MPS }}$ is larger than that of $\Phi_{\text {MPS }} \cdot{ }^{14}$ Various TD-DMRG algorithms ${ }^{74-76}$ approximate the solution to Eq. (10) with different strategies. Here, we apply the so-called tangent-space approach ${ }^{65}$ that replaces the imaginary-time time-dependent Schrödinger equation by the following, projected counterpart

$$
\frac{\partial \Phi_{\mathrm{MPS}}(t)}{\partial t}=-\mathcal{P}_{\Phi_{\mathrm{MPS}}(t)} \mathcal{H} \Phi_{\mathrm{MPS}}(t)
$$

where $\mathcal{P}_{\Phi_{\mathrm{MPS}}(t)}$ is the so-called tangent-space projector ${ }^{64,65}$ which ensures that the bond dimension of the MPS remains constant during the propagation. Lubich and co-workers ${ }^{64}$ derived the following closed-form expression for $\mathcal{P}_{\Phi_{\mathrm{MPS}}(t)}$ :

$$
\mathcal{P}_{\Phi_{\mathrm{MPS}}(t)}=\sum_{i=1}^{L-1}\left(\left|a_{i}^{(l)} \sigma_{i} a_{i+1}^{(r)}\right\rangle\left\langle a_{i}^{(l)} \sigma_{i} a_{i+1}^{(r)}|-| a_{i+1}^{(l)} a_{i+1}^{(r)}\right\rangle\left\langle a_{i+1}^{(l)} a_{i+1}^{(r)}\right|\right)
$$

where $\left|a_{i}^{(l)}\right\rangle$ is the left-renormalized basis for site $i$, defined recursively in terms of $\left|a_{i-1}^{(l)}\right\rangle$ as:

$$
\left|a_{i}^{(l)}\right\rangle=\sum_{a_{i-1} \sigma_{i}} M_{a_{i-1}, a_{i}}^{\sigma_{i}}\left|a_{i-1}^{(l)} \sigma_{i}\right\rangle
$$

and $\left|a_{i}^{(r)}\right\rangle$ is defined analogously. The differential equation obtained by combining Eqs. (12) and (13) can be solved by approximating the resulting time-evolution operator with a secondorder Trotter approximation. ${ }^{65,66}$ Under these approximations, the MPS is propagated for a time step $\Delta t$ by updating the tensors $\boldsymbol{M}^{\sigma_{i}}$ one site after the other in a sweep-like fashion. For each site, the following differential equation is solved:

$$
\frac{\mathrm{d} M_{a_{i-1}, a_{i}}^{\sigma_{i}}}{\mathrm{~d} t}=-\sum_{a_{i-1}^{\prime}, \sigma_{i}^{\prime}, a_{i}^{\prime}} H_{a_{i-1} \sigma_{i} a_{i}, a_{i-1}^{\prime} \sigma_{i}^{\prime} a_{i}^{\prime}} M_{a_{i-1}^{\prime}, a_{i}^{\prime}}^{\sigma_{i}^{\prime}},
$$

where $H_{a_{i-1} \sigma_{i} a_{i}, a_{i-1}^{\prime} \sigma_{i}^{\prime} a_{i}^{\prime}}$ is the representation of the Hamiltonian in the $\left|a_{i}^{(l)} \sigma_{i} a_{i+1}^{(r)}\right\rangle$ basis (referred to in the following as "site basis"). Eq. (15) is a linear differential equation that is solved with Lanczos-based algorithms. The only approximation of our iTD-DMRG approach is therefore the Trotter factorization of the time-evolution operator. This is a remarkable difference with other TD-DMRG formulations that support only short-ranged Hamiltonians ${ }^{74}$ or introduce additional approximations in the solution of the differential equation. ${ }^{75-77}$ 
For real-time evolutions, the propagation of the MPS requires an additional backpropagation step, associated to the second term of Eq. (13). Such step prevents that some components of the MPS are forward propagated twice. However, as discussed by Haegeman and co-workers for spin lattices ${ }^{65}$ and shown by us for vibrational Hamiltonians, ${ }^{66}$ the back-propagation step can be neglected for imaginary-time evolution.

In the following, we will refer to imaginary-time DMRG optimization applied to transcorrelated Hamiltonian, either in real or in momentum space, as tcDMRG, and we will keep the iTD-DMRG acronym for imaginary-time optimization applied to non-transcorrelated, Hermitian Hamiltonians.

In conclusion, we highlight that the right eigenvector of the transcorrelated Hamiltonian can be in principle optimized with the non-Hermitian time-independent DMRG theory introduced by Chan and Van Voorhis in $2005^{78}$ that has been recently extended to classical statistical mechanics. ${ }^{79-81}$ However, iTD-DMRG is a more appealing optimization strategy, mainly for two reasons. First, the diagonalization of the site Hamiltonian with iterative algorithms becomes challenging for non-Hermitian operators. This limited so far the bond dimension that can be targeted to 50-100. Conversely, the solution of the local differential equation of Eq. 15 with iterative schemes ${ }^{82}$ is as complex for non-Hermitian operators as it is for Hermitian ones. Moreover, the non-Hermitian time-independent DMRG theory encodes both the left and right eigenfunctions of the Hamiltonian as MPSs. This is not the case of iTD-DMRG that parametrizes only the right eigenfunction. Alavi and co-workers showed ${ }^{59}$ that the CI representation of the right eigenfunction of the transcorrelated Hamiltonian is much more compact than that of the left one, and we will show in the next section that the same effect is observed in tcDMRG. For this reason, we expect the convergence of nonHermitian TI-DMRG to be much slower than for tcDMRG due to the need of encoding both eigenfunctions as MPS.

\section{MPO representation of the transcorrelated Hamiltonian}

The representation of the Hamiltonian in a given site basis $\left|a_{i}^{(l)} \sigma_{i} a_{i+1}^{(r)}\right\rangle$, required to solve Eq. (15), can be conveniently calculated by encoding $\mathcal{H}_{\mathrm{tc}}$ as matrix product operator $(\mathrm{MPO}):{ }^{14,83,84}$ 


$$
\begin{aligned}
\mathcal{H} & =\sum_{\boldsymbol{\sigma}, \boldsymbol{\sigma}^{\prime}} \sum_{b_{1}, \ldots, b_{L-1}} H_{1, b_{1}}^{\sigma_{1}, \sigma_{1}^{\prime}} H_{b_{1}, b_{2}}^{\sigma_{2}, \sigma_{2}^{\prime}} \cdots H_{b_{L-1}, 1}^{\sigma_{L}, \sigma_{L}^{\prime}}|\boldsymbol{\sigma}\rangle\left\langle\boldsymbol{\sigma}^{\prime}\right| \\
& =\sum_{b_{1}, \ldots, b_{L-1}} \mathcal{H}_{1, b_{1}} \mathcal{H}_{b_{1}, b_{2}} \cdots \mathcal{H}_{b_{L-1}, 1}
\end{aligned}
$$

with

$$
\mathcal{H}_{b_{i-1}, b_{i}}=\sum_{\boldsymbol{\sigma}_{i}, \boldsymbol{\sigma}_{i}^{\prime}} H_{b_{i-1}, b_{i}}^{\sigma_{i}, \sigma_{i}^{\prime}}\left|\boldsymbol{\sigma}_{i}\right\rangle\left\langle\boldsymbol{\sigma}_{i}^{\prime}\right|
$$

(note that we dropped any subscript characterizing the Hamiltonian in the two equations above as the MPO decomposition in this form is general). In Eq. (16), $\mathcal{H}$ is therefore represented as a product of operator-valued matrices $\mathcal{H}_{b_{i-1}, b_{i}}$. Eq. (16) can be interpreted as the operator counterpart of Eq. (11), with the difference that we encode the Hamiltonian exactly as in Eq. (16), while the MPS is an approximation of the exact CI wave function. Several algorithm to construct MPO representations of operators have been proposed in the literature, ${ }^{85-88}$ most of which support only operators with one- and two-body interaction terms. However, they cannot be applied to the momentum-space representation of the Fermi-Hubbard Hamiltonian of Eq. (3) that contains three-body interactions as well. We encode such long-range terms in a compact MPO format by generalizing the algorithm applied by us to electronic ${ }^{22,83}$ and vibrational ${ }^{89,90}$ Hamiltonians, to three-body interaction terms. This algorithm starts from a naive MPO representation of the Hamiltonian $\mathcal{H}$, in which the matrices $\mathcal{H}_{b_{i-1}, b_{i}}$ are diagonal, can then compresses it with so-called "fork" and "merge" operations. Without going into the details of the algorithm that can be found in Ref. 83, a fork operation optimizes the representation of the sum of two operators that share the first second-quantized operator is the same (such as, for instance, $a_{2}^{\dagger} a_{3}$ and $a_{2}^{\dagger} a_{4}$ ). Similarly, a merge operation optimizes the representation of operators strings for which the last second-quantized operator is the same (such as, for instance, $a_{3}^{\dagger} a_{4}$ and $a_{2}^{\dagger} a_{4}$ ). Ref. 83 shows that a particularly compact representation of the Hamiltonian is obtained with two fork and one merge compressions. Following the same idea, we encode three-body terms by applying three fork and two merge compressions.

We highlight that the generality of the algorithm introduced in Refs. 83 enables a straightforward support of three-body interaction terms. This extension would not be as simple within a first-generation DMRG implementation ${ }^{91,92}$ that constructs the representation of 
the Hamiltonian from so-called complementary operators, whose definition is limited to two-body interaction terms and would become very complex for three- and higher-body interaction terms. Even if, as discussed in Ref. 84, the first-generation and MPO/MPS-based formulations of DMRG are formally equivalent, the latter provides a more flexible framework to extend DMRG to transcorrelated Hamiltonians. Moreover, the Fermi-Hubbard Hamiltonian, both in its real- and momentum-space representations, conserves the overall number of $\alpha$ and $\beta$ electrons. We exploit this property to construct a symmetry-adapted MPS ${ }^{93}$ in which the $M_{a_{i-1}, a_{i}}^{\sigma_{i}}$ tensors are block-diagonal and enhance the energy convergence with $m$.

To conclude, we compare the scaling of iTD-DMRG and tcDMRG. In the real-space representation, both Eq. (1) and its transcorrelated counterpart, Eq. (7), contain at most two-body terms. Both operators are therefore represented as MPOs with size scaling as $\mathcal{O}\left(L^{3}\right) \cdot{ }^{94}$ However, as we will show in the following section, the energy convergence with $m$ is comparable for both Hamiltonians, and hence, tcDMRG does not lead to any advantage over iTD-DMRG. Conversely, the momentum-space transcorrelated Fermi-Hubbard Hamiltonian includes three-body terms, and therefore the bond dimension of its MPO representation will scale as $\mathcal{O}\left(L^{5}\right)$. This increases both the computational cost of calculating MPO-MPS contractions and the storage requirements for the boundaries. ${ }^{83}$ As we will discuss in the next section, this higher computational cost will be balanced by a faster energy convergence with $m$. We highlight that the tcDMRG efficiency can be further enhanced by compressing the MPO representation of the transcorrelated Hamiltonian based on the approach presented in Ref. 86. Moreover, all contractions over the $b_{i}$ MPO indexes can be straightforwardly parallelized as suggested in Refs. 95 and 96. The tcDMRG efficiency will strongly benefit from these massively parallelized implementations.

\section{RESULTS}

We applied tcDMRG to the two-dimensional Fermi-Hubbard model for different lattice sizes, fillings, and interaction strengths. If not otherwise specified, we sorted the orbitals in the one-dimensional DMRG lattice with the so-called snake-like ${ }^{97}$ sorting: orbitals corresponding to $i_{x}=0$ were mapped to the first $W$ sites of the lattice, sorted in increasing $i_{y}$ values. Then, the orbitals with $i_{x}=1$ were mapped to sites $W+1, \ldots, 2 W$ with the same sorting, and this procedure was repeated up to $i_{x}=L$. If not otherwise specified, we ran 
time-independent (TI-)DMRG (usually, we would drop the 'TI' label of this standard version for the sake of brevity, but may keep it here in order to avoid confusion), iTD-DMRG, and tcDMRG calculations with the two-site variant that is less prone to converge into local minima of the energy functional than its single-site counterpart. All energies are reported in units of the hopping parameter $t$, and all time-steps are expressed in the corresponding reciprocal units.
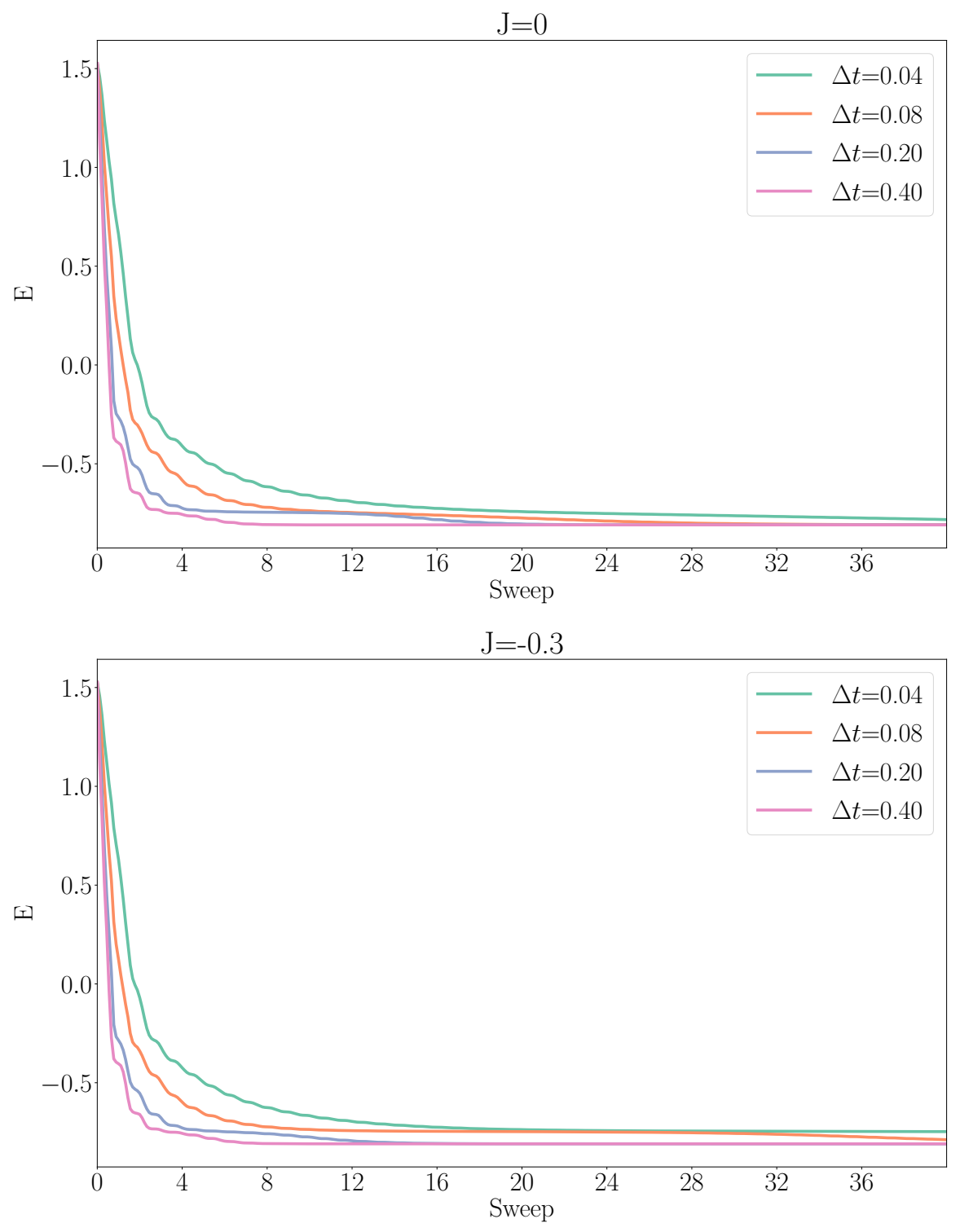

FIG. 1. iTD-DMRG (upper panel) and $\operatorname{tcDMRG}(J=-0.3)$ (lower panel) energy convergence with the sweep number for the real-space $3 \times 3$ Fermi-Hubbard Hamiltonian for varying $\Delta t$ values. The Hamiltonian parameters are $U=8, t=1, \mathrm{~N}_{\alpha}=4, \mathrm{~N}_{\beta}=4$, and $m=300$. 


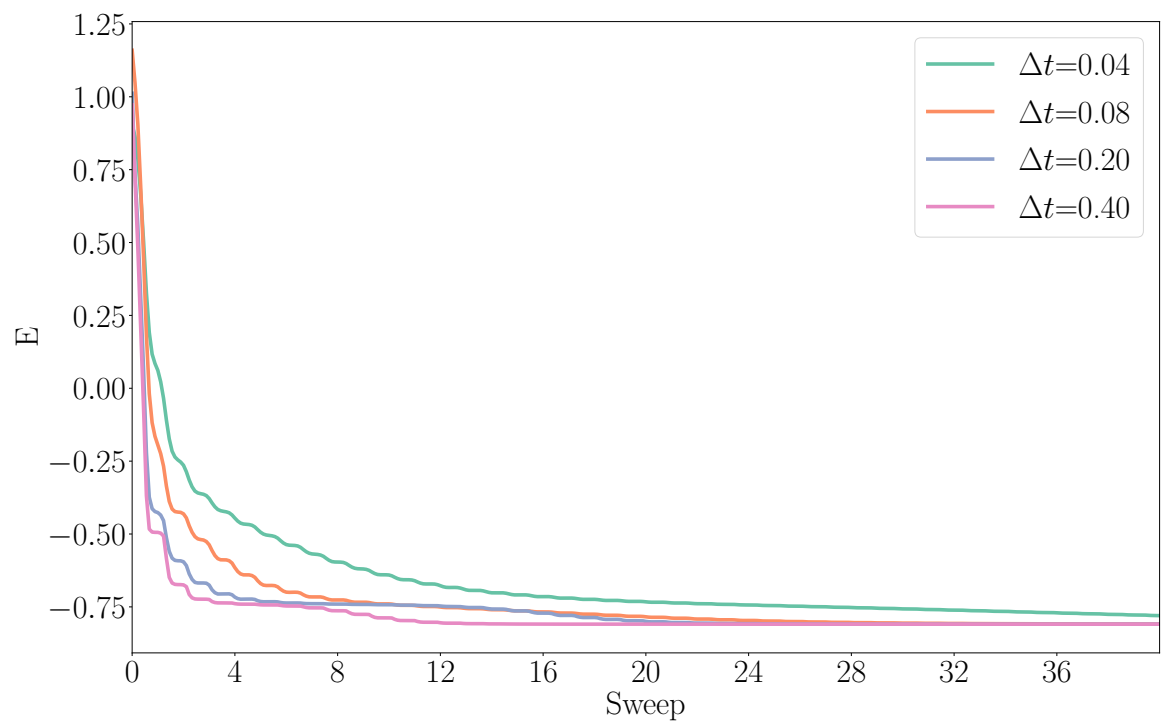

FIG. 2. $\operatorname{tcDMRG}(J=-0.3)$ energy convergence with the sweep number for the $3 \mathrm{x} 3 \boldsymbol{k}$-space FermiHubbard Hamiltonian for varying $\Delta t$ values. The Hamiltonian parameters are $U=8, t=1, \mathrm{~N}_{\alpha}=4$, $\mathrm{N}_{\beta}=4$, and $m=300$.

We first analyze the stability of tcDMRG on a $3 \times 3$ lattice with $U=8$ and $t=1, N_{\alpha}=4$ and $N_{\beta}=4$. We report in Figure 1 the energy convergence of iTD-DMRG and tcDMRG $(J=-$ $0.3)$ for varying time steps for the real-space representation with $m=300$. As we will show in the following, this $m$ value delivers converged energies. Both iTD-DMRG and tcDMRG converge smoothly, the faster convergence being obtained with the largest time step, of 0.40. For a fixed sweep number, larger time steps corresponds to longer overall propagation times, which enables to reach the $t \rightarrow+\infty$ limit faster. Note, however, that the computational cost of solving the local differential equation of Eq. (15) increases with the time step because a larger number of iterations is required to converge the iterative approximation of the exponential operator. For $\Delta t=0.40$, the Lanczos approximation of the local representation of the imaginary-time propagator converges within 15 iterations in all cases. These results confirm that, unlike TI-DMRG, imaginary-time TD-DMRG can reliably optimize the ground-state wave function of non-Hermitian Hamiltonians.

As we show in Figure 2, the same trend is observed for the $\boldsymbol{k}$-space Fermi-Hubbard Hamiltonian. The fastest convergence (12 sweeps) is observed with $\Delta t=0.4$ au. With smaller time steps, the energy converges to a local minimum between sweeps 10 and 20, and only 
afterwards the algorithm converges to the correct limit. For this reason, if not otherwise stated, in the following we set $\Delta t=0.40$ for all calculations.

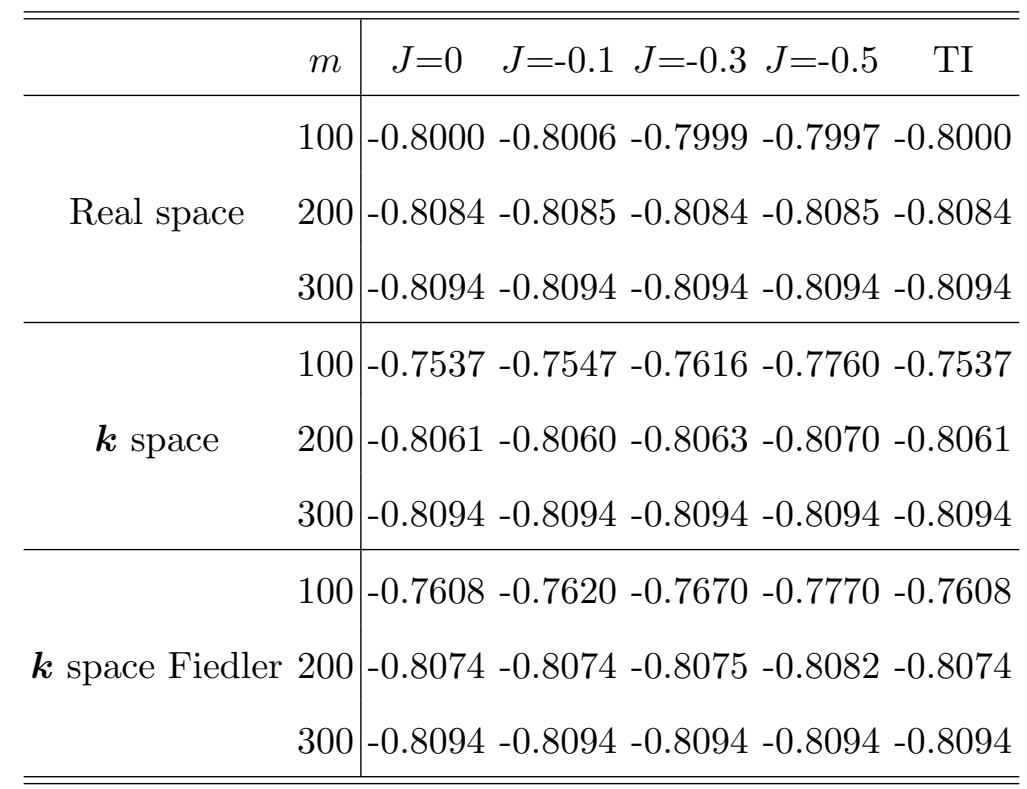

TABLE I. Ground-state energy per site of the 3x3 Fermi-Hubbard model obtained with iTD-DMRG and tcDMRG, $U=8, t=1, N_{\alpha}=4$, and $N_{\beta}=4$ for varying bond dimension $m$. The reference energy per site, obtained with exact diagonalization, ${ }^{98}$ is -0.8094 . TI-DMRG energies are reported in the last column.

We depict in Table I the TI-DMRG, iTD-DMRG, and tcDMRG energy convergence with bond dimension $m$ and the transcorrelation parameter $J$ for both Hamiltonian representation. As expected, all methods converge towards the reference full-CI energy ${ }^{98}$ with $m=300$. Note that this $m$ value is large for a 9-orbital system, and this is due to the breakdown of the area law for long-ranged Hamiltonians, as we already noted in Section II. As highlighted by Ref. 97, the average interaction range of the Hamiltonian can be strongly reduced by optimizing the orbital sorting in the one-dimensional DMRG lattice based on the so-called Fiedler ordering. ${ }^{99}$ This orbital sorting minimizes the distance between strongly interacting orbitals, where the interaction strength is evaluated from the mutual information ${ }^{100,101}$ calculated for the MPS optimized with a partially converged MPS, here obtained with TIDMRG with $m=100$. Note that in Ref. 97 the impact of the ordering on the wave function entanglement is analyzed only qualitatively. Here we assess its effect also on the energy convergence with the bond dimension $m$. As expected, we show in Table I, the energy for a given $m$ value is consistently lower with the Fiedler ordering than with the standard one. 
We report in Table I tcDMRG results obtained with different $J$ values. For the realspace Fermi-Hubbard Hamiltonian, the tcDMRG energy matches the TI-DMRG one for all $J$ values for $m=200$ and 300. With $m=100$, the energy difference between TI-DMRG and tcDMRG is smaller than 0.0006 in all cases. The transcorrelated ansatz of Eq. (4) does not produce therefore a more compact MPS when applied to the real-space two-dimensional Fermi-Hubbard Hamiltonian. This agrees with the fact that in Ref. 59 Alavi and coworkers applied the transcorrelated FCIQMC algorithm to the $\boldsymbol{k}$-space two-dimensional Fermi-Hubbard Hamiltonian, and they did not present any results for the real-space representation.

The energy difference between TI-DMRG and tcDMRG is instead considerable for the $\boldsymbol{k}$-space representation. In this case, the difference between the $\operatorname{tcDMRG}(J=-0.5)$ energies obtained with $m=100$ and $m=300$ is nearly halved compared to the corresponding TI-DMRG data. Note that a correlation factor of $J=-0.5$ is similar to the values optimized in Ref. 59 for lattices with similar $U / t$ values. This confirms that the ground-state wave function of the 3x3 Fermi-Hubbard Hamiltonian can be encoded as a much more compact MPS with the addition of a Gutzwiller correlator.

\begin{tabular}{|c|c|c|c|c|c|}
\hline & $m$ & $J=0$ & $J=-0.1 \quad J=-0.3 \quad J=-0.5$ & $J=-0.5$ Fiedler & TI \\
\hline & 500 & -1.0248 & $-1.0249-1.0255-1.0269$ & -1.0260 & -1.0249 \\
\hline $\boldsymbol{k}$-space & 1000 & -1.0282 & $-1.0281-1.0284-1.0285$ & -1.0283 & -1.0283 \\
\hline & 2000 & -1.0288 & $-1.0288-1.0288-1.0288$ & -1.0285 & -1.0288 \\
\hline
\end{tabular}

TABLE II. Ground-state energy per site for the $4 \times 4$ Fermi-Hubbard model with $U=8, t=1, N_{\alpha}=4$, and $N_{\beta}=4$. The reference energy, obtained with exact diagonalization, ${ }^{98}$ is -1.0288 .

We report in Table II the iTD-DMRG and tcDMRG results for a larger, $4 \mathrm{x} 4$ lattice with $U=8, t=1, N_{\alpha}=4$, and $N_{\beta}=4$, a parameter set that corresponds to an intermediate correlation regime. The energy convergence with $m$ is slower than for the $3 \times 3$ lattice, and the TI-DMRG energy matches the reference value ${ }^{98}$ with $m=2000$. Also in this case, the energy convergence with $m$ is faster for tcDMRG than for iTD-DMRG. The difference between $m=500$ and fully-converged tcDMRG $(J=-0.5)$ energies is twice as small than for TI-DMRG. The lowest energy, for a given $m$ value, is consistently obtained with $\operatorname{tcDMRG}(J=-0.5)$ and the Fiedler orbital sorting. 
We have applied so-far tcDMRG to either small or weakly-correlated Hamiltonians, for which iTD-DMRG, when combined with an optimized orbital sorting, can converge the energy with reasonable $m$ values. The efficiency of tcDMRG becomes apparent in the strong correlation regime, such as for the $4 \times 4$ Fermi-Hubbard Hamiltonian with $U=4, t=1, N_{\alpha}=8$, and $N_{\beta}=8$. We report in Table III the corresponding TI-DMRG, iTD-DMRG, and tcDMRG energies for varying $J$ and $m$ values and different orbital sortings. To avoid convergence into local minima, we adopted the so-called density-matrix perturbation theory approach by White ${ }^{102}$ extended to a two-site optimizer for all TI-DMRG simulations. The computational cost of this perturbative scheme is high, especially for large $m$ values, and would render tcDMRG calculations unpractical. To avoid a large computational overhead, we adopted the following protocol: we optimized the wave function with TI-DMRG and $m=500$ adding the density-matrix perturbation. Then, we started TI-DMRG optimizations for larger $m$ values with the resulting MPS as starting guess. Finally, we ran all tcDMRG calculations for a given $m$ values with the MPS optimized with TI-DMRG and the same $m$ value as initial guess.

\begin{tabular}{|c|c|c|}
\hline & $m$ & $J=-0.1 \quad J=-0.3 \quad J=-0.5$ \\
\hline \multirow{3}{*}{$\boldsymbol{k}$-space } & 500 & $-0.7900-0.7779-0.8496-0.7862$ \\
\hline & 1000 & 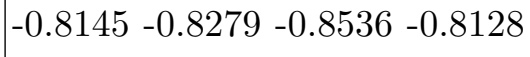 \\
\hline & 2000 & 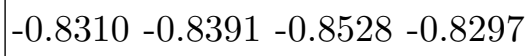 \\
\hline \multirow{4}{*}{$\boldsymbol{k}$-space Fiedler } & 500 & $-0.8485-0.8495-0.8515-0.8484$ \\
\hline & 1000 & 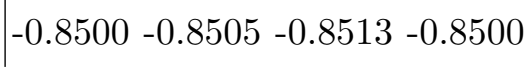 \\
\hline & 2000 & $-0.8507-0.8511-0.8514-0.8507$ \\
\hline & 500 Herm. & 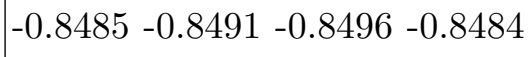 \\
\hline
\end{tabular}

TABLE III. Ground-state energy per site for a $4 \mathrm{x} 4$ Fermi-Hubbard model with $U=4, t=1, N_{\alpha}=8$, and $N_{\beta}=8$. The reference energy per site, obtained with exact diagonalization, ${ }^{98}$ is -0.8514 .

As expected, the energy convergence of TI-DMRG with $m$ is much slower than for the previous Hamiltonian, and the $m=2000$ energy $(-0.8297)$ is still far from being converged to the reference result (-0.8514) ${ }^{98}$ The energy convergence of tcDMRG with the bond dimension $m$ is, however, much faster. The faster convergence is delivered by $\operatorname{tcDMRG}(J=-0.5)$ that converges below 0.001 with $m=2000$. 


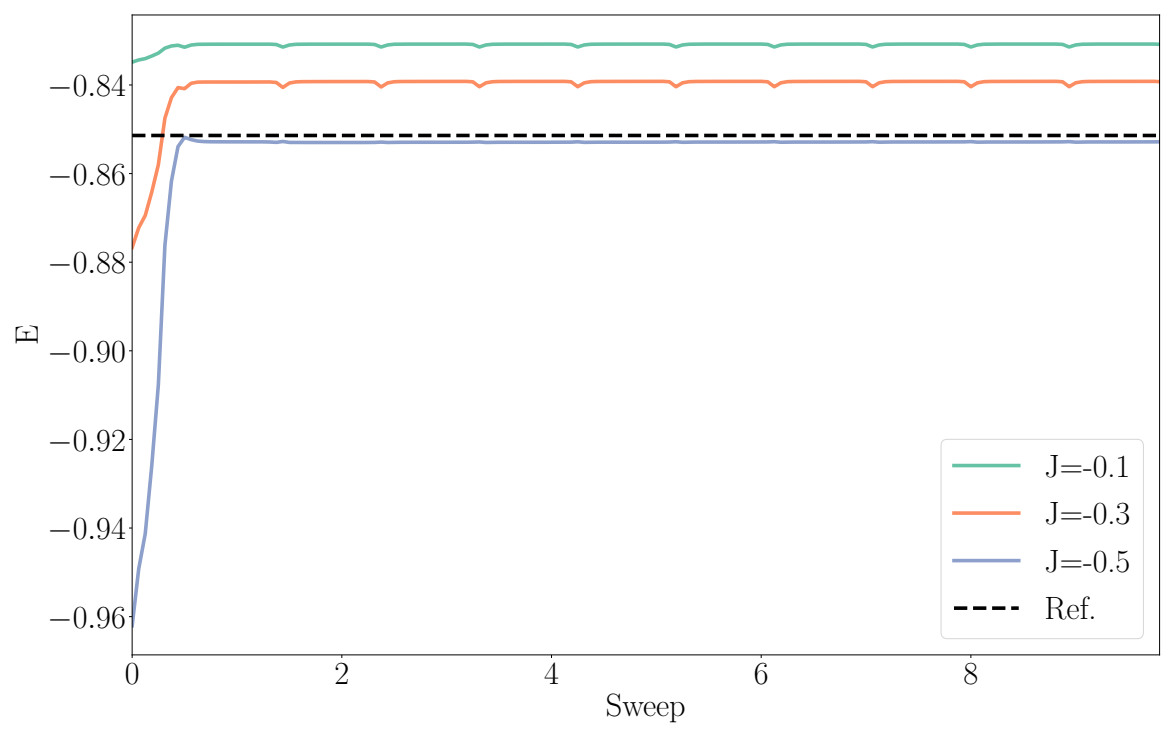

FIG. 3. tcDMRG energy convergence for the $4 \mathrm{x} 4$ Fermi-Hubbard Hamiltonian with $U / t=4, N_{\alpha}=8$, $N_{\beta}=8$, for different $J$ values. The initial guess is obtained from the MPS optpimized with TIDMRG in all cases. The reference energy, taken from Ref. 98, is reported as well.

As we show in Figure 3, our computational procedure that starts the tcDMRG imaginarytime propagation from the MPS optimized with TI-DMRG converges the energy efficiently with 2-3 sweeps. It is also worth noting that the energy is lower than the reference, fullCI value for $J=0.3$ and $J=0.5$. We recall that the energy is the expectation value of the transcorrelated Hamiltonian (see Eq. (8)) over the MPS and, as we already highlighted in Section II, the variational principle does not apply. Therefore, an energy lower than the exact, full-CI one is physically acceptable in this case. We highlight that, unlike tcDMRG, a variational-based optimization starting from the TI guess would not converge to the correct minimum. We conclude by noting that the $J$ value that provides the best match with the reference data is 0.5. This value agrees with the optimal value obtained in Ref. 59 based on the optimization strategy described in Ref. 67 for a 18-sites Fermi-Hubbard Hamiltonian with the same $U / t$ value. This suggest that the same algorithm, ${ }^{67}$ that requires the solution of a Coupled Cluster-like equation, can be effectively applied to determine the optimal $J$ value for tcDMRG. Note that the results reported in Figure 3 also indicate that the transcorrelation parameter $J$ cannot be optimized variationally.

As we show in Table III, the orbital sorting has a critical impact in the energy convergence 
of DMRG. With the Fiedler ordering, the TI-DMRG energy obtained with $m=500$ is only 0.0023 higher than the reference energy, while the same difference with the Fiedler ordering is larger than 0.04. However, even by adopting the Fiedler ordering, the energy is not converged even with $m=2000$. By combining the optimized orbital ordering with tcDMRG $(J=-0.5)$, the energy differs from the reference one by only $10^{-4}$ already with $m=500$. Therefore, the energy convergence of tcDMRG with the bond dimension is in this case truly faster than that of TI-DMRG. This further confirms that, also in the presence of strong correlation, the ground state of the Fermi-Hubbard model can be parametrized as Eq. (4), where $|\Phi\rangle$ can be efficiently represented as a low-entanglement wave function and optimized by applying iTD-DMRG to the transcorrelated Hamiltonian.

Alavi and co-workers ${ }^{59}$ showed that, if, the right lowest-energy eigenvector of the transcorrelated Hamiltonian can be represented as compact CI wave functions, the left one will be represented by a much less compact expansion. Similarly, as we show in the last row of Table III, the convergence of the energy of the left eigenvector is slower than for the right one (as discussed in Ref. 59, the right-eigenvector corresponding to a given $J$ value can be optimized with tcDMRG by setting the Jastrow factor to $-J$ ).

To further characterize the entanglement structure of the ground-state wave function of the transcorrelated Hamiltonian, we report in Figure 4 a graphical representation of the orbital mutual information matrix $I_{i j},{ }^{100,101,103}$ with

$$
I_{i j}=\frac{1}{2}\left[s_{i}(1)+s_{j}(1)-s_{i j}(2)\right]\left(1-\delta_{i j}\right)
$$

calculated with TI-DMRG and $\operatorname{tcDMRG}(J=-0.5)$ for different orbital orderings. $s_{i}(1)$ is the single-orbital entropy for orbital $i$, defined as

$$
s_{i}(1)=-\sum_{\alpha=1}^{4} w_{i, \alpha} \ln w_{i, \alpha}
$$

where $w_{i, \alpha}$ is the $\alpha$-th eigenvalue of the one-orbital reduced density matrix. Similarly, $s_{i j}(2)$ is the two-orbital entropy, defined as

$$
s_{i j}(2)=-\sum_{\alpha=1}^{16} w_{i j, \alpha} \ln w_{i j, \alpha}
$$

where $w_{i j, \alpha}$ the $\alpha$-th eigenvalue of the two-orbital reduced density matrix for orbitals $i$ and j. As expected, the mutual information matrix has a sparse structure with the standard 

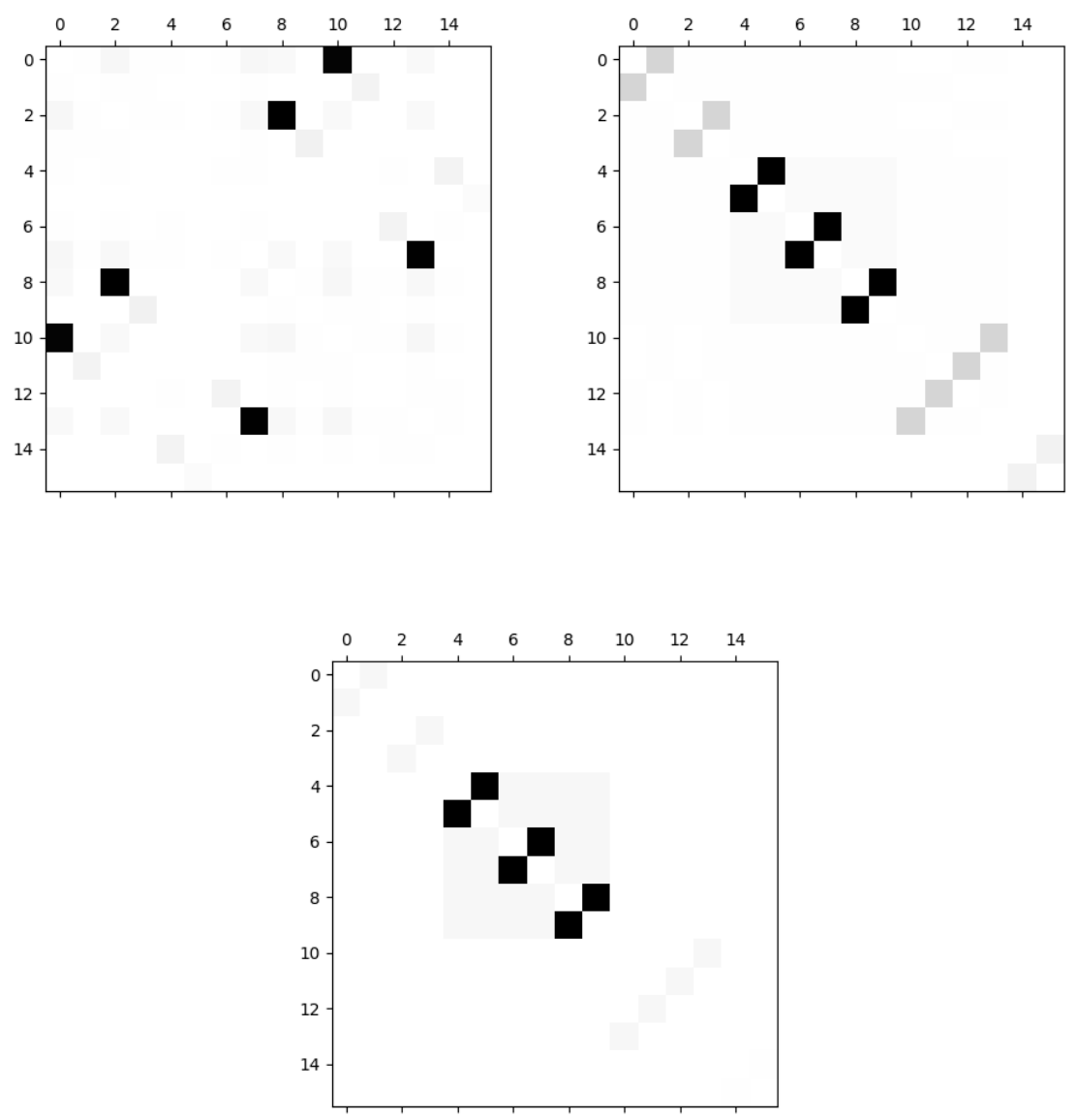

FIG. 4. Graphical representation of the mutual information matrix $I_{i j}$ for a $4 \times 4$ Fermi-Hubbard lattice with $U / t=4$ at half filling, obtained with TI-DMRG based on the standard ordering (upper left), TI-DMRG with the Fiedler ordering (upper right), and tcDMRG with the Fiedler ordering (bottom).

ordering, while it becomes diagonally dominant for the MPS constructed with the Fiedler ordering. Most importantly, the magnitude of most non-zero elements of the TI-DMRG mutual information matrix are much smaller for $\operatorname{tcDMRG}(J=-0.5)$. This further confirms that the multi-reference character, measured as orbital entanglement, of the ground state of the transcorrelated Hamiltonian is much smaller than that of the original Fermi-Hubbard Hamiltonian. For this reason, the former can be much more efficiently represented as an MPS. We recall that a similar regularization of the orbital mutual information has been previously observed when combining DMRG with short-range DFT (DMRG-srDFT). ${ }^{41}$ In DMRG-srDFT the Hamiltonian is modified to include only the long-range part of the electron-electron 
Coulomb interaction. Similarly, tcDMRG removes dynamical correlation with the Jastrow factorization of the wave function. In both cases, the mutual information associated to the DMRG wave function becomes more sparse since it is large only for the orbitals coupled by pure static correlation effects.

\begin{tabular}{cccc|cc|c}
\hline \hline$U / t$ & $\mathrm{~N}_{\alpha}$ & $\mathrm{N}_{\beta}$ & $m$ & \multicolumn{2}{|c|}{ TI-DMRG tcDMRG } & Ref. \\
\hline \hline 4 & 12 & 12 & 500 & -1.1500 & -1.1804 & -1.1853 \\
2 & 18 & 18 & 500 & -1.1345 & -1.1530 & -1.1516 \\
4 & 18 & 18 & 500 & -0.8206 & -0.8596 & -0.8574 \\
4 & 18 & 18 & 1000 & -0.8307 & -0.8580 & -0.8574 \\
\hline \hline
\end{tabular}

TABLE IV. TI-DMRG and tcDMRG ground-state energy per site of a 6x6 Fermi-Hubbard model with different number of electrons and interaction strengths, for varying $m$ values. The reference benchmark data are taken from Refs. 98 and 104 and are calculated with auxiliary field Quantum Monte Carlo.

We report in Table IV the TI-DMRG and tcDMRG energies of the 6x6 Fermi-Hubbard lattice, a system that has been studied with the transcorrelated variant of FCIQMC ${ }^{59}$ and which is out of the reach for exact-diagonalization approaches, for various fillings and $U / t$ ratios. To limit the computational cost, we obtained the tcDMRG results with the single-site imaginary-time propagator starting from the MPS optimized with TI-DMRG. In all cases, we sorted the orbitals in the lattice with the Fiedler ordering obtained with TI-DMRG $(m=500)$. Moreover, we set the parameter $J$ to the optimal value taken from Ref. 59. The results reported in Table IV confirm that, also for large lattices, the energy convergence with $m$ of tcDMRG is much faster than for standard TI-DMRG. In all cases, the difference between TI-DMRG $(m=500)$ and the reference energies is larger than 0.02. The error becomes one order of magnitude smaller with $\operatorname{tcDMRG}(m=500)$, and is consistently lower than 0.004 . By further increasing $m$ to 1000, the energy of the half-filled $6 x 6$ lattice with $U / t=4$, which is the most strongly correlated lattice among the ones studied here, the tcDMRG energy matches the reference one with an error smaller than $10^{-3}$. 


\section{CONCLUSIONS}

In this work, we introduced transcorrelated DMRG theory that optimizes the groundstate wave function of the transcorrelated Hamiltonian as a matrix product state. The optimization algorithm is tailored to the problem: imaginary-time time-dependent DMRG. Unlike standard time-independent DMRG relying on the variational principle, iTD-DMRG can reliably optimize the ground-state energy of non-Hermitian Hamiltonians. We applied tcDMRG to the two-dimensional Fermi-Hubbard Hamiltonian for different sizes, fillings, and interaction strengths. We demonstrate that, both for weak and strong correlation regimes, the energy convergence of tcDMRG is consistently much faster than that of TIDMRG. In practice, tcDMRG can converge the energy of Fermi-Hubbard lattices including up to 36 sites, which otherwise would be a challenge for standard DMRG approaches due to the constraints imposed by the area law. Our results agree with recent findings in the framework of FCIQMC by Alavi and co-workers, who showed that the ground-state of the electronic ${ }^{60}$ and Fermi-Hubbard ${ }^{59}$ transcorrelated Hamiltonians are efficiently represented by very compact configuration-interaction expansions. In addition, we showed in the present work that the ground-state wave functions belong, more generally, to the class of lowentanglement wave functions and can be therefore encoded as a compact MPS. Note that this conclusion extends the findings of Alavi and co-workers ${ }^{59}$ since a compact MPS wave function can encode both sparse and dense full-CI expansions.

The successful application of the tcDMRG algorithm to the two-dimensional FermiHubbard Hamiltonian suggests that the same theory can be applied as successfully to the electronic Hamiltonian as well. ${ }^{60}$ In this case, the Jastrow factor in conveniently expressed in real space because the similarity-transformed Hamiltonian includes exactly only up to three-body terms, the main hurdle being the need of calculating the resulting threecenters integrals. However, we expect that tcDMRG will be even more efficient when applied to electronic-structure problems for two reasons. In fact, the Gutzwiller correlator of the transcorrelated Fermi-Hubbard Hamiltonian is governed by a single parameter. Conversely, the real-space Jastrow factor includes a different correlation parameter for each orbital pair. The wave function parametrization is, therefore, much more flexible and can be adapted to the specific molecular system under analysis. Moreover, the three-body potential term appearing in the transcorrelated Fermi-Hubbard Hamiltonian couples any possible combi- 
nation of six different orbitals. The MPO representation of the resulting Hamiltonian is highly non-compact, even though in the present work we discussed a way to encode it efficiently. For the quantum-chemical case, it will be possible to exploit orbital locality to screen and compress the three-body part of the Hamiltonian and encode it as a compact MPO and to further enhance the tcDMRG efficiency. We showed that the similarity transformation underlying tcDMRG regularizes the wave function entanglement and makes the distinction between strongly- and weakly-correlated orbitals more rigid. This suggests that tcDMRG can be effectively combined with active-space based approaches, where dynamical correlation effects are added a-posteriori with perturbation theory or with multi-reference variants of the Coupled-Cluster method. ${ }^{105-108}$ All these extensions are currently explored in our laboratory and results will be reported in future work.

\section{ACKNOWLEDGMENTS}

This work was supported by ETH Zürich through the ETH Fellowship No. FEL-49 18-1.

\section{REFERENCES}

${ }^{1}$ G. H. Booth, A. J. W. Thom, and A. Alavi, "Fermion Monte Carlo without fixed nodes: A game of life, death, and annihilation in Slater determinant space," J. Chem. Phys. 131, 54106 (2009).

${ }^{2}$ D. Cleland, G. H. Booth, and A. Alavi, "Communications: Survival of the fittest: Accelerating convergence in full configuration-interaction quantum Monte Carlo," J. Chem. Phys. 132, 041103 (2010).

${ }^{3}$ B. Huron, J. P. Malrieu, and P. Rancurel, "Iterative perturbation calculations of ground and excited state energies from multiconfigurational zeroth-order wave functions," J. Chem. Phys. 58, 5745-5759 (1973).

${ }^{4}$ S. Evangelisti, J.-P. Daudey, and J.-P. Malrieu, "Convergence of an improved CIPSI algorithm," Chem. Phys. 75, 91-102 (1983).

${ }^{5}$ T. Zhang and F. A. Evangelista, "A Deterministic Projector Configuration Interaction Approach for the Ground State of Quantum Many-Body Systems," J. Chem. Theory Comput. 12, 4326-4337 (2016). 
${ }^{6}$ N. M. Tubman, J. Lee, T. Y. Takeshita, M. Head-Gordon, and K. B. Whaley, "A deterministic alternative to the full configuration interaction quantum Monte Carlo method," J. Chem. Phys. 145, 044112 (2016).

${ }^{7}$ J. J. Eriksen, F. Lipparini, and J. Gauss, "Virtual Orbital Many-Body Expansions: A Possible Route towards the Full Configuration Interaction Limit," J. Phys. Chem. Lett. 8, 4633-4639 (2017).

${ }^{8}$ S. R. White, "Density matrix formulation for quantum renormalization groups," Phys. Rev. Lett. 69, 2863-2866 (1992).

${ }^{9}$ S. R. White, "Density-matrix algorithms for quantum renormalization groups," Phys. Rev. B 48, 10345-10356 (1993).

${ }^{10}$ Ö. Legeza, R. M. Noack, J. Sólyom, and L. Tincani, "Applications of quantum information in the density-matrix renormalization group," Lect. Notes Phys. 739, 653-664 (2008).

${ }^{11}$ G. K.-L. Chan, J. J. Dorando, D. Ghosh, J. Hachmann, E. Neuscamman, H. Wang, and T. Yanai, "An Introduction to the Density Matrix Renormalization Group Ansatz in Quantum Chemistry," in Frontiers in Quantum Systems in Chemistry and Physics (Springer Netherlands, 2008) pp. 49-65.

${ }^{12}$ G. K. L. Chan and D. Zgid, "The Density Matrix Renormalization Group in Quantum Chemistry," Annual Reports in Computational Chemistry 5, 149-162 (2009).

${ }^{13} \mathrm{~K}$. H. Marti and M. Reiher, "The density matrix renormalization group algorithm in quantum chemistry," Z. Phys. Chem. 224, 583-599 (2010).

${ }^{14} \mathrm{U}$. Schollwöck, "The density-matrix renormalization group in the age of matrix product states," Ann. Phys. 326, 96-192 (2011).

${ }^{15}$ G. K.-L. Chan and S. Sharma, "The Density Matrix Renormalization Group in Quantum Chemistry," Annu. Rev. Phys. Chem. 62, 465-481 (2011).

${ }^{16} \mathrm{~S}$. Wouters and D. Van Neck, "The density matrix renormalization group for ab initio quantum chemistry," Eur. Phys. J. D 31, 395-402 (2013).

${ }^{17}$ Y. Kurashige, "Multireference electron correlation methods with density matrix renormalisation group reference functions," Mol. Phys. 112, 1485-1494 (2014).

${ }^{18}$ R. Olivares-Amaya, W. Hu, N. Nakatani, S. Sharma, J. Yang, and G. K.-L. Chan, "The ab-initio density matrix renormalization group in practice," J. Chem. Phys. 142, 34102 (2015). 
${ }^{19}$ S. Szalay, M. Pfeffer, V. Murg, G. Barcza, F. Verstraete, R. Schneider, and Ö. Legeza, "Tensor product methods and entanglement optimization for ab initio quantum chemistry," Int. J. Quantum Chem. 115, 1342-1391 (2015).

${ }^{20}$ T. Yanai, Y. Kurashige, W. Mizukami, J. Chalupský, T. N. Lan, and M. Saitow, "Density matrix renormalization group for ab initio calculations and associated dynamic correlation methods: A review of theory and applications," Int. J. Quantum Chem. 115, 283-299 (2015).

${ }^{21}$ S. Knecht, E. D. Hedegård, S. Keller, A. Kovyrshin, Y. Ma, A. Muolo, C. J. Stein, and M. Reiher, "New Approaches for ab initio Calculations of Molecules with Strong Electron Correlation," Chimia 70, 244-251 (2016).

${ }^{22}$ A. Baiardi and M. Reiher, "The density matrix renormalization group in chemistry and molecular physics: Recent developments and new challenges," J. Chem. Phys. 152, 040903 (2020).

${ }^{23}$ K. T. Williams, Y. Yao, J. Li, L. Chen, H. Shi, M. Motta, C. Niu, U. Ray, S. Guo, R. J. Anderson, J. Li, L. N. Tran, C.-N. Yeh, B. Mussard, S. Sharma, F. Bruneval, M. van Schilfgaarde, G. H. Booth, G. K.-L. Chan, S. Zhang, E. Gull, D. Zgid, A. Millis, C. J. Umrigar, and L. K. Wagner, "Direct Comparison of Many-Body Methods for Realistic Electronic Hamiltonians," Phys. Rev. X 10, 011041 (2020).

${ }^{24}$ J. J. Eriksen, T. A. Anderson, J. E. Deustua, K. Ghanem, D. Hait, M. R. Hoffmann, S. Lee, D. S. Levine, I. Magoulas, J. Shen, N. M. Tubman, K. B. Whaley, E. Xu, Y. Yao, N. Zhang, A. Alavi, G. K.-L. Chan, M. Head-Gordon, W. Liu, P. Piecuch, S. Sharma, S. L. Ten-no, C. J. Umrigar, and J. Gauss, "The Ground State Electronic Energy of Benzene," J. Phys. Chem. Lett. 11, 8922-8929 (2020).

${ }^{25}$ Y. Kurashige and T. Yanai, "Second-order perturbation theory with a density matrix renormalization group self-consistent field reference function: Theory and application to the study of chromium dimer," J. Chem. Phys. 135, 94104 (2011).

${ }^{26}$ S. Sharma and G. K.-L. Chan, "A flexible multi-reference perturbation theory by minimizing the Hylleraas functional with matrix product states," J. Chem. Phys. 141, 111101 (2014).

${ }^{27}$ Y. Kurashige, J. Chalupský, T. N. Lan, and T. Yanai, "Complete active space secondorder perturbation theory with cumulant approximation for extended active-space wavefunction from density matrix renormalization group," J. Chem. Phys. 141, 174111 (2014). 
${ }^{28}$ M. Roemelt, S. Guo, and G. K.-L. Chan, "A projected approximation to strongly contracted N-electron valence perturbation theory for DMRG wavefunctions," J. Chem. Phys. 144, $204113(2016)$.

${ }^{29}$ J. Ren, Y. Yi, and Z. Shuai, "Inner Space Perturbation Theory in Matrix Product States: Replacing Expensive Iterative Diagonalization," J. Chem. Theory Comput. 12, 4871-4878 (2016).

${ }^{30}$ S. Wouters, V. Van Speybroeck, and D. Van Neck, "DMRG-CASPT2 study of the longitudinal static second hyperpolarizability of all-trans polyenes," J. Chem. Phys. 145, 54120 (2016).

${ }^{31}$ S. Guo, M. A. Watson, W. Hu, Q. Sun, and G. K.-L. Chan, "N -Electron Valence State Perturbation Theory Based on a Density Matrix Renormalization Group Reference Function, with Applications to the Chromium Dimer and a Trimer Model of Poly( p -Phenylenevinylene)," J. Chem. Theory Comput. 12, 1583-1591 (2016).

${ }^{32}$ S. Sharma, G. Jeanmairet, and A. Alavi, "Quasi-degenerate perturbation theory using matrix product states," J. Chem. Phys. 144, 34103 (2016).

${ }^{33}$ L. Freitag, S. Knecht, C. Angeli, and M. Reiher, "Multireference Perturbation Theory with Cholesky Decomposition for the Density Matrix Renormalization Group," J. Chem. Theory Comput. 13, 451-459 (2017).

${ }^{34}$ S. Sharma, G. Knizia, S. Guo, and A. Alavi, "Combining Internally Contracted States and Matrix Product States To Perform Multireference Perturbation Theory," J. Chem. Theory Comput. 13, 488-498 (2017).

${ }^{35}$ S. Guo, Z. Li, and G. K.-L. Chan, "Communication: An efficient stochastic algorithm for the perturbative density matrix renormalization group in large active spaces," J. Chem. Phys. 148, 221104 (2018).

${ }^{36}$ S. Sharma, A. A. Holmes, G. Jeanmairet, A. Alavi, and C. J. Umrigar, "Semistochastic Heat-Bath Configuration Interaction Method: Selected Configuration Interaction with Semistochastic Perturbation Theory," J. Chem. Theory Comput. 13, 1595-1604 (2017).

${ }^{37}$ Y. Garniron, A. Scemama, P. F. Loos, and M. Caffarel, "Hybrid stochastic-deterministic calculation of the second-order perturbative contribution of multireference perturbation theory," J. Chem. Phys. 147, 034101 (2017).

${ }^{38}$ A. Mahajan, N. S. Blunt, I. Sabzevari, and S. Sharma, "Multireference configuration interaction and perturbation theory without reduced density matrices," J. Chem. Phys. 
151, $211102(2019)$.

${ }^{39}$ J. Toulouse, F. Colonna, and A. Savin, "Long-range - Short-range separation of the electron-electron interaction in density-functional theory," Phys. Rev. A 70, 62505 (2004).

${ }^{40}$ G. Li Manni, R. K. Carlson, S. Luo, D. Ma, J. Olsen, D. G. Truhlar, and L. Gagliardi, "Multiconfiguration pair-density functional theory," J. Chem. Theory Comput. 10, 36693680 (2014).

${ }^{41}$ E. D. Hedegård, S. Knecht, J. S. Kielberg, H. J. Aagaard Jensen, and M. Reiher, "Density matrix renormalization group with efficient dynamical electron correlation through range separation," J. Chem. Phys. 142, 224108 (2015).

${ }^{42}$ E. Giner, B. Pradines, A. Ferté, R. Assaraf, A. Savin, and J. Toulouse, "Curing basis-set convergence of wave-function theory using density-functional theory: A systematically improvable approach,” J. Chem. Phys. 149, 194301 (2018).

${ }^{43}$ P. Sharma, V. Bernales, S. Knecht, D. G. Truhlar, and L. Gagliardi, "Density matrix renormalization group pair-density functional theory (DMRG-PDFT): singlet-triplet gaps in polyacenes and polyacetylenes," Chem. Sci. 10, 1716-1723 (2019).

${ }^{44} \mathrm{~S}$. Ten-no, "Initiation of explicitly correlated Slater-type geminal theory," Chem. Phys. Lett. 398, 56-61 (2004).

${ }^{45}$ W. Klopper, F. R. Manby, S. Ten-No, and E. F. Valeev, "R12 methods in explicitly correlated molecular electronic structure theory," Int. Rev. Phys. Chem.. 25, 427-468 (2006).

${ }^{46}$ L. Kong, F. A. Bischoff, and E. F. Valeev, "Explicitly correlated R12/F12 methods for electronic structure," Chem. Rev. 112, 75-107 (2012).

${ }^{47}$ C. Hättig, W. Klopper, A. Köhn, and D. P. Tew, "Explicitly correlated electrons in molecules," Chem. Rev. 112, 4-74 (2012).

${ }^{48}$ C. Hättig, D. P. Tew, and A. Köhn, "Communications: Accurate and efficient approximations to explicitly correlated coupled-cluster singles and doubles, CCSD-F12," J. Chem. Phys. 132, 231102 (2010).

${ }^{49}$ Q. Ma and H.-J. Werner, "Explicitly correlated local coupled-cluster methods using pair natural orbitals," Wiley Interdiscip. Rev. Comput. Mol. Sci. 8, e1371 (2018).

${ }^{50}$ T. Shiozaki and H. J. Werner, "Communication: Second-order multireference perturbation theory with explicit correlation: CASPT2-F12," J. Chem. Phys. 133, 141103 (2010).

${ }^{51}$ T. Shiozaki and H. J. Werner, "Multireference explicitly correlated F12 theories," Mol. 
Phys. 111, 607-630 (2013).

${ }^{52}$ S. F. Boys and N. C. Handy, "The Determination of Energies and Wavefunctions with Full Electronic Correlation," Proc. R. Soc. A Math. Phys. Eng. Sci. 310, 43-61 (1969).

${ }^{53}$ N. C. Handy, "Towards an understanding of the form of correlated wavefunctions for atoms," J. Chem. Phys. 58, 279-287 (1973).

${ }^{54}$ R. Jastrow, "Many-body problem with strong forces," Phys. Rev. 98, 1479-1484 (1955).

${ }^{55}$ T. Yanai and G. K.-L. Chan, "Canonical transformation theory for multireference problems," J. Chem. Phys. 124, 194106 (2006).

${ }^{56}$ H. Luo, "Complete optimisation of multi-configuration Jastrow wave functions by variational transcorrelated method," J. Chem. Phys. 135, 024109 (2011).

${ }^{57}$ H. Luo, "Variational transcorrelated method," J. Chem. Phys. 133, 154109 (2010).

${ }^{58}$ H. Luo and A. Alavi, "Combining the Transcorrelated Method with Full Configuration Interaction Quantum Monte Carlo: Application to the Homogeneous Electron Gas," J. Chem. Theory Comput. 14, 1403-1411 (2018).

${ }^{59}$ W. Dobrautz, H. Luo, and A. Alavi, "Compact numerical solutions to the two-dimensional repulsive Hubbard model obtained via nonunitary similarity transformations," Phys. Rev. B 99, 075119 (2019).

${ }^{60}$ A. J. Cohen, H. Luo, K. Guther, W. Dobrautz, D. P. Tew, and A. Alavi, "Similarity transformation of the electronic Schrödinger equation via Jastrow factorization," J. Chem. Phys. 151, 061101 (2019).

${ }^{61}$ M. Motta, T. P. Gujarati, J. E. Rice, A. Kumar, C. Masteran, J. A. Latone, E. Lee, E. F. Valeev, and T. Y. Takeshita, "Quantum simulation of electronic structure with transcorrelated Hamiltonian: increasing accuracy without extra quantum resources," ArXiv eprints , 2006.02488 (2020).

${ }^{62} \mathrm{~S}$. McArdle and D. P. Tew, "Improving the accuracy of quantum computational chemistry using the transcorrelated method," ArXiv e-prints , 2006.11181 (2020).

${ }^{63}$ S. Paeckel, T. Köhler, A. Swoboda, S. R. Manmana, U. Schollwöck, and C. Hubig, "Time-evolution methods for matrix-product states," Ann. Phys. 411, 167998 (2019).

${ }^{64}$ C. Lubich, I. Oseledets, and B. Vandereycken, "Time integration of tensor trains," SIAM J. Numer. Anal. 53, 917 (2015).

${ }^{65}$ J. Haegeman, C. Lubich, I. Oseledets, B. Vandereycken, and F. Verstraete, "Unifying time evolution and optimization with matrix product states," Phys. Rev. B 94, 165116 
(2016).

${ }^{66}$ A. Baiardi and M. Reiher, "Large-scale quantum-dynamics with matrix product states," J. Chem. Theory Comput. 15, 3481-3498 (2019).

${ }^{67}$ J. M. Wahlen-Strothman, C. A. Jiménez-Hoyos, T. M. Henderson, and G. E. Scuseria, "Lie algebraic similarity transformed Hamiltonians for lattice model systems," Phys. Rev. B 91, 041114 (2015), arXiv:1409.2203.

${ }^{68}$ S. Tsuneyuki, "Transcorrelated Method: Another Possible Way towards Electronic Structure Calculation of Solids," Prog. Theor. Phys. Supp. 176, 134-142 (2008).

${ }^{69}$ E. Neuscamman, H. Changlani, J. Kinder, and G. K. L. Chan, "Nonstochastic algorithms for Jastrow-Slater and correlator product state wave functions," Phys. Rev. B 84, 205132 (2011).

${ }^{70}$ E. Stoudenmire and S. R. White, "Studying Two-Dimensional Systems with the Density Matrix Renormalization Group," Ann. Rev. Cond. Matt. Phys. 3, 111-128 (2012).

${ }^{71}$ J. Motruk, M. P. Zaletel, R. S. Mong, and F. Pollmann, "Density matrix renormalization group on a cylinder in mixed real and momentum space," Phys. Rev. B 93, 155139 (2016), arXiv:1512.03318.

${ }^{72}$ G. Ehlers, S. R. White, and R. M. Noack, "Hybrid-space density matrix renormalization group study of the doped two-dimensional Hubbard model," Phys. Rev. B 95, 125125 (2017).

${ }^{73}$ M. B. Hastings, "An area law for one-dimensional quantum systems," J. Stat. Mech. Theory Exp. 2007, P08024-P08024 (2007).

${ }^{74}$ V. Guifre, "Efficient simulation of one-dimensional quantum many-body systems," Phys. Rev. Lett. 93, 40501-40502 (2004).

${ }^{75}$ K. A. Al-Hassanieh, A. E. Feiguin, J. A. Riera, C. A. Büsser, and E. Dagotto, "Adaptive time-dependent density-matrix renormalization-group technique for calculating the conductance of strongly correlated nanostructures," Phys. Rev. B 73, 195304 (2006).

${ }^{76}$ E. Ronca, Z. Li, C. A. Jimenez-Hoyos, and G. K. L. Chan, "Time-Step Targeting TimeDependent and Dynamical Density Matrix Renormalization Group Algorithms with ab Initio Hamiltonians," J. Chem. Theory Comput. 13, 5560-5571 (2017).

${ }^{77}$ L.-H. Frahm and D. Pfannkuche, "Ultrafast ab-initio Quantum Chemistry Using Matrix Product States," J. Chem. Theory Comput. 15, 2154-2165 (2019).

${ }^{78}$ G. K. L. Chan and T. Van Voorhis, "Density-matrix renormalization-group algorithms 
with nonorthogonal orbitals and non-Hermitian operators, and applications to polyenes," J. Chem. Phys. 122, 204101 (2005).

${ }^{79}$ E. Carlon, M. Henkel, and U. Schollwöck, "Density matrix renormalization group and reaction-diffusion processes," Eur. Phys. J. B 12, 99-114 (1999).

${ }^{80}$ P. Helms, U. Ray, and G. K. L. Chan, "Dynamical phase behavior of the single- and multi-lane asymmetric simple exclusion process via matrix product states," Phys. Rev. E 100, 022101 (2019).

${ }^{81}$ P. Helms and G. K.-L. Chan, "Dynamical Phase Transitions in a 2D Classical Nonequilibrium Model via 2D Tensor Networks," Phys. Rev. Lett. 125, 140601 (2020).

${ }^{82}$ Y. Saad, "Analysis of Some Krylov Subspace Approximations to the Matrix Exponential Operator," SIAM J. Numer. Anal. 29, 209-228 (1992).

${ }^{83}$ S. Keller, M. Dolfi, M. Troyer, and M. Reiher, "An efficient matrix product operator representation of the quantum chemical Hamiltonian," J. Chem. Phys 143, 244118 (2015).

${ }^{84}$ G. K.-l. Chan, A. Keselman, N. Nakatani, Z. Li, and S. R. White, "Matrix product operators, matrix product states, and ab initio density matrix renormalization group algorithms," J. Chem. Phys. 145, 014102 (2016).

${ }^{85}$ B. Pirvu, V. Murg, J. I. Cirac, and F. Verstraete, "Matrix product operator representations," New J. Phys. 12, 25012 (2010).

${ }^{86}$ F. Fröwis, V. Nebendahl, and W. Dür, "Tensor operators: Constructions and applications for long-range interaction systems," Phys. Rev. A 81, 62337 (2010).

${ }^{87}$ C. Hubig, I. P. McCulloch, and U. Schollwöck, "Generic construction of efficient matrix product operators," Phys. Rev. B 95, 35129 (2017).

${ }^{88}$ J. Ren, W. Li, T. Jiang, and Z. Shuai, "A general automatic method for optimal construction of matrix product operators using bipartite graph theory," J. Chem. Phys. 153, $084118(2020)$.

${ }^{89}$ A. Baiardi, C. J. Stein, V. Barone, and M. Reiher, "Vibrational Density Matrix Renormalization Group," J. Chem. Theory Comput. 13, 3764-3777 (2017).

${ }^{90}$ A. Baiardi, C. J. Stein, V. Barone, and M. Reiher, "Optimization of highly excited matrix product states with an application to vibrational spectroscopy," J. Chem. Phys. 150, 094113 (2019).

${ }^{91} \mathrm{~S}$. R. White and R. L. Martin, "Ab initio quantum chemistry using the density matrix renormalization group," J. Chem. Phys. 110, 4127-4130 (1999). 
${ }^{92}$ G. K.-L. Chan and M. Head-Gordon, "Highly correlated calculations with a polynomial cost algorithm: A study of the density matrix renormalization group," J. Chem. Phys. 116, 4462-4476 (2002).

${ }^{93}$ S. Singh, R. N. C. Pfeifer, and G. Vidal, "Tensor network states and algorithms in the presence of a global U(1) symmetry," Phys. Rev. B 83, 115125 (2011).

${ }^{94}$ M. Dolfi, B. Bauer, S. Keller, A. Kosenkov, T. Ewart, A. Kantian, T. Giamarchi, and M. Troyer, "Matrix product state applications for the ALPS project," Comput. Phys. Commun. 185, 3430-3440 (2014).

${ }^{95}$ A. Kantian, M. Dolfi, M. Troyer, and T. Giamarchi, "Understanding repulsively mediated superconductivity of correlated electrons via massively parallel density matrix renormalization group," Phys. Rev. B 100, 75138 (2019).

${ }^{96}$ J. Brabec, J. Brandejs, K. Kowalski, S. Xantheas, Ö. Legeza, and L. Veis, "Massively parallel quantum chemical density matrix renormalization group method," ArXiv e-prints , $2001.04890(2020)$.

${ }^{97}$ G. Ehlers, J. Sólyom, O. Legeza, and R. M. Noack, "Entanglement structure of the Hubbard model in momentum space," Phys. Rev. B 92, 235116 (2015).

${ }^{98}$ H. Shi and S. Zhang, "Symmetry in auxiliary-field quantum Monte Carlo calculations," Phys. Rev. B 88, 125132 (2013).

${ }^{99}$ G. Barcza, Ö. Legeza, K. H. Marti, and M. Reiher, "Quantum-information analysis of electronic states of different molecular structures," Phys. Rev. A 83, 012508 (2011).

${ }^{100}$ Ö. Legeza and J. Sólyom, "Optimizing the density-matrix renormalization group method using quantum information entropy," Phys. Rev. B 68, 195116 (2003).

${ }^{101}$ J. Rissler, R. M. Noack, and S. R. White, "Measuring orbital interaction using quantum information theory," Chem. Phys. 323, 519-531 (2006).

${ }^{102}$ S. R. White, "Density matrix renormalization group algorithms with a single center site," Phys. Rev. B 72, 180403 (2005).

${ }^{103}$ C. J. Stein and M. Reiher, "autoCAS: A Program for Fully Automated Multiconfigurational Calculations," J. Comput. Chem. 40, 2216 (2019).

${ }^{104}$ M. Qin, H. Shi, and S. Zhang, "Benchmark study of the two-dimensional Hubbard model with auxiliary-field quantum Monte Carlo method," Phys. Rev. B 94, 085103 (2016).

${ }^{105}$ X. Li, G. Peris, J. Planelles, F. Rajadall, and J. Paldus, "Externally corrected singles and doubles coupled cluster methods for open-shell systems," J. Chem. Phys. 107, 90-98 
(1997).

${ }^{106}$ T. Kinoshita, O. Hino, and R. J. Bartlett, "Coupled-cluster method tailored by configuration interaction," J. Chem. Phys. 123, 074106 (2005).

${ }^{107}$ L. Veis, A. Antalík, J. Brabec, F. Neese, Ö. Legeza, and J. Pittner, "Coupled Cluster Method with Single and Double Excitations Tailored by Matrix Product State Wave Functions," J. Phys. Chem. Lett. 7, 4072-4078 (2016).

${ }^{108}$ F. A. Evangelista, "Perspective : Multireference coupled cluster theories of dynamical electron correlation," J. Chem. Phys. 149, 030901 (2018). 